\title{
CONJUGACY IN PERMUTATION REPRESENTATIONS OF THE SYMMETRIC GROUP
}

\author{
YONA CHERNIAVSKY AND MISHAEL SKLARZ
}

\begin{abstract}
Although the conjugacy classes of the general linear group are known, it is not obvious (from the canonic form of matrices) that two permutation matrices are similar if and only if they are conjugate as permutations in the symmetric group, i.e. that conjugacy classes of $S_{n}$ do not unite under the natural representation. We prove this fact, and give its application to the enumeration of fixed points under a natural action of $S_{n} \times S_{n}$. We also consider the permutation representations of $S_{n}$ which arise from the action of $S_{n}$ on $k$-tuples, and classify which of them unite conjugacy classes and which do not.
\end{abstract}

\section{INTRODUCTION}

In this paper we study the action of $S_{n}$ on $k$-tuples. Denote by $\rho_{k}$ the corresponding permutation representation over an arbitrary field $\mathbb{F}$. The following problem was presented to us by Lubotzky and Roichman.

Problem 1. For which $1 \leq k \leq n$ and for which fields $\mathbb{F}$ does the following hold:

For any two permutations $\pi, \sigma \in S_{n}, \rho_{k}(\pi)$ is conjugate to $\rho_{k}(\sigma)$ in $G L(n, \mathbb{F})$ if and only if $\pi$ and $\sigma$ are conjugate in $S_{n}$.

This problem arises in the enumeration of invertible matrices with respect to a certain natural action of $S_{n} \times S_{n}$, see $[\mathrm{BC}]$ and Section 4 below.

For $k=n$, i.e. the regular representation, a negative solution to Problem 1 was essentially given by Burnside (see B p. 23-24). In Section 2 it is shown that for $k=1$ the answer is positive. A full solution is given in Section 3 We find that $\rho_{1}$ and $\rho_{2}$ do not unite any classes, that $\rho_{3}$ unites classes only when $n$ is even, and that $\rho_{k}$ for $k \geq 4$ always unites some classes. These results do not depend on the choice of the field $\mathbb{F}$. Finally, our results are applied in Section 4 to the enumeration of fixed points of a natural action of $S_{n} \times S_{n}$ on invertible matrices.

\section{The Natural Representation of $S_{n}$}

There is an obvious embedding of $S_{n}$ in $G L(n, \mathbb{F})$ where $\mathbb{F}$ is any field. Consider a permutation $\pi \in S_{n}$ as an $n \times n$ matrix obtained from the identity matrix by permutations of the rows. More explicitly: for every permutation $\pi \in S_{n}$ we identify $\pi$ with the matrix:

2000 Mathematics Subject Classification. Primary 20C30; Secondary 05E15.

Key words and phrases. conjugacy classes, symmetric group, permutation representations, characters, fixed points. 


$$
[\pi]_{i, j}=\left\{\begin{array}{cc}
1 & i=\pi(j) \\
0 & \text { otherwise }
\end{array}\right.
$$

This representation can also be realized as the permutation representation which is obtained from the natural action of $S_{n}$ on $\{1,2, \ldots, n\}$ defined by $\pi \cdot i=\pi(i)$.

Our first result is that this representation does not unite conjugacy classes of $S_{n}$. We shall use the following well known fact:

Fact 2.1. If $\sigma$ is a cycle of length $n$, then $\sigma^{k}$ consists of $(n, k)$ cycles, each of length $n /(n, k){ }^{1}$

Theorem 2.2. The conjugacy classes of $S_{n}$ do not unite in $G L(n, \mathbb{F})$. In other words, if $\pi$ and $\sigma$ are permutations with similar matrices in $G L(n, \mathbb{F})$, then they are conjugate in $S_{n}$ too.

Proof. Let $\pi$ and $\sigma$ be permutations which are similar as matrices. First of all, we note that for any $k, \pi^{k}$ and $\sigma^{k}$ are also similar.

Case 1: $\operatorname{char}(\mathbb{F})=0$ or at least $\operatorname{char}(\mathbb{F})>n$.

Each cycle of length $k$ in $\pi$ contributes the term $x^{k}-1$ into the characteristic polynomial of the permutation matrix. Under the above restriction on $\operatorname{char}(\mathbb{F})$ it seems reasonable that the cycle structure of a permutation can be recovered from the characteristic polynomial of the corresponding permutation matrix. However, our proof utilizes the trace of the permutation matrix and the traces of its powers.

Denote by $c_{d}(\pi)$ the number cycles with length equal to $d$ in $\pi$. We shall use induction on $d$ to prove that $c_{d}(\pi)=c_{d}(\sigma)$, for all $d$, and this will show that $\pi$ and $\sigma$ are conjugate.

Since $\pi$ and $\sigma$ are similar as matrices, we have $\operatorname{trace}(\pi)=\operatorname{trace}(\sigma)$. However, the trace function counts the 1's on the diagonal (here we use the restriction on $\operatorname{char}(\mathbb{F})$ ), and each such 1 corresponds to a fixed point of the permutation, so $\operatorname{trace}(\pi)=c_{1}(\pi)$. Therefore, $c_{1}(\pi)=c_{1}(\sigma)$, i.e. $\pi$ and $\sigma$ have the same number of fixed points. This is the base of our induction.

Now let $d$ be an arbitrary number, and suppose that $c_{k}(\pi)=c_{k}(\sigma)$ for all $k<d$. From Lemma 2.1 it follows that a $k$-cycle in $\pi$ ends up as a product of $k 1$-cycles in $\pi^{d}$ if and only if $k$ divides $d$. Therefore, we can conclude that

$$
\operatorname{trace}\left(\pi^{d}\right)=\sum_{k \mid d} k \cdot c_{k}(\pi)=d \cdot c_{d}(\pi)+\sum_{k \mid d, k<d} k \cdot c_{k}(\pi) .
$$

Now, by our induction hypothesis, for all proper divisors $k \mid d$ we have $c_{k}(\pi)=$ $c_{k}(\sigma)$. On the other hand, $\operatorname{trace}\left(\pi^{d}\right)=\operatorname{trace}\left(\sigma^{d}\right)$. This implies that $c_{d}(\pi)=c_{d}(\sigma)$, and completes the induction argument.

We have shown that $\pi$ and $\sigma$ have the same cycle structure, so they are conjugate as permutations.

Case 2: With no restriction on $\operatorname{char}(\mathbb{F})$

In this case, the trace of a permutation matrix no longer gives the number of fixed points of the permutation, so a more devious route is necessary.

Note that in this case it is impossible to recover the cycle structure of a permutation from the characteristic polynomial of the corresponding permutation matrix:

\footnotetext{
${ }^{1}$ We use $(n, k)$ to denote the greatest common divisor of $n$ and $k$.
} 
for example, if $\operatorname{char}(\mathbb{F})=2$ we have $x^{4}+1=\left(x^{2}+1\right)^{2}=(x+1)^{4}$, i.e. one cycle of length 4 , two cycles of length 2 and four cycles of length 1 have the same characteristic polynomial.

Claim 2.3. Denote by $m(\pi)$ the number of cycles in $\pi . m(\pi)$ is invariant for similar matrices, i.e., $m(\pi)=m(\sigma)$

Proof. The number 1 is an eigenvalue of all permutation matrices, because $(1,1, \ldots, 1)^{T}$ is an eigenvector. Since $\pi$ acts on vectors by permuting their coordinates, solving the equation $\pi \underline{x}=\underline{x}$ (which is the equation to find eigenvecors of the eigenvalue 1 ) we have the following: each cycle $\left(i_{1}, i_{2}, \ldots, i_{k}\right)$ in $\pi$ gives us $k$ equations on corresponding entries of $\underline{x}$

$$
x_{i_{k}}=x_{i_{1}}, x_{i_{1}}=x_{i_{2}}, \ldots, x_{i_{k-1}}=x_{i_{k}}
$$

which means that all these entries are equal. Thus we have that the number of free variables, which is the dimension of 1-eigenspace, equal to the number of cycles in $\pi$. Noting that the dimension of an eigenspace is invariant for similar matrices proves our point.

Claim 2.4. Denote by $m_{d}(\pi)$ the number of cycles in $\pi$ with length divisible by $d$. $m_{d}(\pi)$ is also invariant for similar matrices, i.e., $m_{d}(\pi)=m_{d}(\sigma)$

Proof. First, take $d=2$ as an example. Any even-length cycle in $\pi$ splits into two cycles in $\pi^{2}$, while the odd cycles stay unsplit. This means that $m_{2}(\pi)=$ $m\left(\pi^{2}\right)-m(\pi)=m\left(\sigma^{2}\right)-m(\sigma)=m_{2}(\sigma)$. More generally, for any prime number $p$, the cycles of length divisible by $p$ split into $p$ separate cycles, while cycles of length non-divisible by $p$ remain unsplit. Therefore,

$$
m_{p}(\pi)=\frac{m\left(\pi^{p}\right)-m(\pi)}{p-1},
$$

which implies that $m_{p}(\pi)=m_{p}(\sigma)$ by Claim 2.3

Now turn to the proof in general case.

We shall proceed by induction on the length of the prime decomposition of $d$. First, for $d=1, m_{d}(\pi)=m(\pi)$, and our claim follows from Claim 2.3

Suppose that $d=p t$, with $p$ prime. An appeal to Lemma 2.1 shows that cycles in $\pi$ of length divisible by $p t$ split into $p$ separate cycles in $\pi^{p}$, each of length divisible by $t$. The cycles in $\pi$ whose length is non-divisible by $p t$ either split into cycles of length non-divisible by $t$ or don't split at all. Working backwards, a cycle in $\pi^{p}$ of length divisible by $t$ either comes from a cycle in $\pi$ that was divisible by $p t$ (in which case it will be one of $p$ such cycles), or comes from a cycle in $\pi$ of length divisible by $t$ but not by $p$.

If $t$ is prime to $p$, the number of cycles in $\pi$ divisible by $t$ but not by $p$ is $\left(m_{t}(\pi)-m_{p t}(\pi)\right)$, so we have $m_{t}\left(\pi^{p}\right)=p \cdot m_{p t}(\pi)+\left(m_{t}(\pi)-m_{p t}(\pi)\right)$. On the other hand, if $t$ is divisible by $p$, there are no cycles divisible by $t$ but not by $p$, so we have $m_{p t}(\pi)=p \cdot m_{t}\left(\pi^{p}\right)$. In summary, we have shown that

$$
m_{p t}(\pi)= \begin{cases}\frac{m_{t}\left(\pi^{p}\right)-m_{t}(\pi)}{p-1} & (p, t)=1 \\ \frac{m_{t}\left(\pi^{p}\right)}{p} & (p, t)>1\end{cases}
$$

Now, $t$ has a shorter prime decomposition, so by our induction hypothesis, $m_{t}$ is invariant, so $m_{t}(\pi)=m_{t}(\sigma)$, and $m_{t}\left(\pi^{p}\right)=m_{t}\left(\sigma^{p}\right)$. This shows that $m_{n}(\pi)=$ $m_{n}(\sigma)$, as was to be shown. 
A short example is in place. Suppose $\pi$ has cycle decomposition $\left[3^{2}, 6^{2}, 9,12^{2}\right]$. Then $\pi^{2}$ has decomposition $\left[3^{6}, 6^{4}, 9\right]$, and $\pi^{3}$ decomposes as $\left[1^{6}, 2^{6}, 3^{3}, 4^{6}\right]$. On the one hand, we have

$$
m_{12}(\pi)=m_{3 \cdot 4}(\pi)=\left(m_{4}\left(\pi^{3}\right)-m_{4}(\pi)\right) /(3-1)=(6-2) / 2=2 .
$$

On the other hand,

$$
m_{12}(\pi)=m_{2 \cdot 6}(\pi)=m_{6}\left(\pi^{2}\right) / 2=4 / 2=2,
$$

and we see that both calculations give us the expected results.

Claim 2.5. $c_{d}(\pi)$ (the number of cycles of exact length $d$ ) is also invariant, so $c_{d}(\pi)=c_{d}(\sigma)$.

Proof. Fix some permutation $\pi$, and let $I_{d}$ denote the set of cycles of length divisible by $d$ in $\pi$. Evidently $m_{d}(\pi)=\left|I_{d}\right|$. Also, we have $I_{d} \cap I_{k}=I_{[d, k]}$, so $\left|I_{d} \cap I_{k}\right|=$ $m_{[d, k]}(\pi)$. The same goes for intersections of more than two such sets. Now, it is also clear that $c_{d}(\pi)=\left|I_{d}-\cup_{k>1} I_{d k}\right|=\left|I_{d}\right|-\left|\cup_{k>1} I_{d k}\right|$. Using the inclusion-exclusion principal, we end up with a sum

$$
c_{d}(\pi)=\left|I_{d}\right|-\sum_{k>1}\left|I_{d k}\right|+\sum_{k_{1}, k_{2}>1}\left|I_{d k_{1}} \cap I_{d k_{2}}\right|-\ldots
$$

and as we saw, all the summands are in fact of the form $m_{k}(\pi)$, for some $k$. In the previous claim we showed that all the $m_{k}$ are invariant, so $c_{d}$ is invariant too, and our claim is proved.

Again, a short example might clarify things. Using $\pi$ from the example above, with cycle type $\left[3^{2}, 6^{2}, 9,12^{2}\right]$, we have

$$
\begin{aligned}
c_{3}(\pi)= & \left|I_{3}\right|-\left(\left|I_{6}\right|+\left|I_{9}\right|+\left|I_{12}\right|\right)+\left(\left|I_{6} \cap I_{9}\right|+\left|I_{6} \cap I_{12}\right|+\left|I_{9} \cap I_{12}\right|\right) \\
& -\left|I_{6} \cap I_{9} \cap I_{12}\right| \\
= & \left|I_{3}\right|-\left(\left|I_{6}\right|+\left|I_{9}\right|+\left|I_{12}\right|\right)+\left(\left|I_{18}\right|+\left|I_{12}\right|+\left|I_{36}\right|\right)-\left|I_{36}\right| \\
= & m_{3}-\left(m_{6}+m_{9}+m_{12}\right)+\left(m_{18}+m_{12}+m_{36}\right)-m_{36} \\
= & 7-(4+1+2)+(0+2+0)-0=2
\end{aligned}
$$

and that indeed is the correct result.

Summing up, Claim 2.5 means that permutation matrices $\pi$ and $\sigma$ which are similar as matrices have the same cycle structure, i.e. are conjugate in the symmetric group and thus the proof of Theorem 2.2 is completed.

\section{Other Permutation Representations}

As we mentioned above, the natural representation of $S_{n}$ can be realized as the permutation representation obtained from the natural action of $S_{n}$ on $\{1,2, \ldots, n\}$, defined by $\pi \cdot i=\pi(i)$. We now consider the permutation representation $\rho_{k}^{\mathbb{F}}$, which arise from the action of $S_{n}$ on $k$-tuples, where the action is defined by $\pi \cdot\left(i_{1}, \ldots, i_{k}\right)=\left(\pi\left(i_{1}\right), \ldots, \pi\left(i_{k}\right)\right)$. These representations form an interpolation between the natural representation (1-tuples) and the regular representation ( $n$ tuples). 
3.1. Representations Over the Complex Field. In Section 2 we proved that the natural representation of $S_{n}$ does not unite conjugacy classes. On the other hand, it is well known ([B] p. 23-24) that the regular representation of $S_{n}$ (indeed, of any group) unites all elements of equal order. The natural representation can be seen as the permutation representation obtained from the natural action of $S_{n}$ on the set $\{(1),(2), \ldots,(n)\}$ of 1-tuples. On the other hand, the regular representation can be seen as the permutation representation which arises from the action of $S_{n}$ on all $n$ ! ordered $n$-tuples of numbers from $\{1,2, \ldots, n\}$. In this section we wish to address the representations in between: the representation arising from the action of $S_{n}$ on pairs, triplets, etc. and to see where the representations start uniting conjugacy classes.

We begin with a general result, which holds true for any representation of any finite group.

Theorem 3.1. Let $G$ be a group, and $\sigma, \tau \in G$. Let $T: G \rightarrow G L(d, \mathbb{C})$ a representation of $G$, with character $\chi$. Then $T(\sigma) \sim T(\tau)$ as matrices if and only if $\chi\left(\sigma^{k}\right)=\chi\left(\tau^{k}\right)$ for all $k$.

Proof. Obviously, if $T(\sigma) \sim T(\tau)$, then $T\left(\sigma^{k}\right) \sim T\left(\tau^{k}\right)$ for any $k$, so $\chi\left(\sigma^{k}\right)=$ $\operatorname{trace}\left(T\left(\sigma^{k}\right)\right)=\operatorname{trace}\left(T\left(\tau^{k}\right)\right)=\chi\left(\tau^{k}\right)$.

Now suppose that $\chi\left(\sigma^{k}\right)=\chi\left(\tau^{k}\right)$ for all $k$. Denote $s=|\sigma|$ and $t=|\tau|$, and let $m=[s, t]$. Now consider $C=\langle x\rangle$, a cyclic group or order $m$, and define two representations of $C: T_{\sigma}(x)=T(\sigma)$ and $T_{\tau}(x)=T(\tau)$. It is easily seen that these are indeed well defined representations. The hypothesis now reads that these two representations have the same character, and so they must be similar [CR]. This implies that $T(\sigma) \sim T(\tau)$.

Note that the fact that the regular representation unites all elements of equal order can be derived from this theorem: If $\chi$ is the character of the regular representation, then

$$
\chi\left(\sigma^{k}\right)= \begin{cases}|G| & |\sigma| \mid k \\ 0 & \text { otherwise }\end{cases}
$$

so obviously $\chi\left(\sigma^{k}\right)=\chi\left(\tau^{k}\right)$ for all $k$ if and only if $\sigma$ and $\tau$ have the same order.

We also note that representations which are not faithful trivially unite all the conjugacy classes in their kernel with 1 . We may therefore omit them from our discussion from now on. Faithful representations can only unite two classes if their elements have the same order.

The criterion which we just presented is still rather complicated to use for general groups, but it can be simplified in our case, because of the following simple fact.

Fact 3.2. Let $\sigma \in S_{n}$, with $|\sigma|=m$.

- If $k$ is relatively prime to $m$. Then $\sigma^{k} \sim \sigma$.

- For any $k, \sigma^{k} \sim \sigma^{(m, k)}$.

Claim 3.3. Let $T: S_{n} \rightarrow G L(d, \mathbb{C})$ be a representation of the symmetric group, with character $\chi$, and $\sigma, \tau \in S_{n}$ elements of order $m$. Then $T(\sigma) \sim T(\tau)$ if and only if $\chi\left(\sigma^{k}\right)=\chi\left(\tau^{k}\right)$ for $k \mid m$.

Proof. If $T(\sigma) \sim T(\tau)$, then obviously $\chi\left(\sigma^{k}\right)=\chi\left(\tau^{k}\right)$ for any $k$, and in particular for divisors of $m$. On the other hand, suppose $\chi\left(\sigma^{k}\right)=\sigma\left(\tau^{k}\right)$ for $k \mid m$. Now, for any $k, \sigma^{k} \sim \sigma^{(k, m)}$, and similarly for $\tau$. Therefore, using the fact that $(k, m) \mid m$, 
$\chi\left(\sigma^{k}\right)=\chi\left(\sigma^{(k, m)}\right)=\chi\left(\sigma^{(k, m)}\right)=\chi\left(\tau^{k}\right)$. This holds for all $k$, and therefore, by Theorem 3.1 $T(\sigma) \sim T(\tau)$.

Corrolary 3.4. If $\sigma$ and $\tau$ are of prime order $p$, then $T(\sigma) \sim T(\tau)$ if and only if $\chi(\sigma)=\chi(\tau)$.

Definition 3.5. Let $\sigma, \tau \in S_{n}$ be elements of equal order $m$, such that if $k \neq 1$ and $k \mid m$ then $\sigma^{k} \sim \tau^{k}$. It follows from 3.3 that $T(\sigma) \sim T(\tau)$ if and only if $\chi(\sigma)=\chi(\tau)$. We call such elements almost similar. In fact, it is sufficient to require that $\sigma^{p} \sim \tau^{p}$ for all prime divisors of $m$.

We next show that almost similar elements are typical examples of elements that are united by representations, in the following sense:

Theorem 3.6. Let $T: S_{n} \rightarrow G L(d, \mathbb{C})$ be a representation. If $T$ unites some two conjugacy classes, then there must exist a pair of almost similar elements which it unites.

Proof. Suppose that $\sigma$ and $\tau$ are non-similar elements such that $T(\sigma) \sim T(\tau)$. Obviously $T\left(\sigma^{k}\right) \sim T\left(\tau^{k}\right)$, so it suffices to show that there exists some $k$ for which $\sigma^{k}$ and $\tau^{k}$ are almost similar but non-similar. We show this by induction on the number of primes in the prime decomposition of $m$, the order of $\sigma$ and $\tau$. If $m$ is of length 1, i.e. $m$ is prime, then $\sigma$ and $\tau$ already are almost similar. In general, if $\sigma^{p} \sim \tau^{p}$ for all prime divisors $p \mid m$, then $\sigma$ and $\tau$ are already almost similar. Otherwise, there exists some prime $p \mid m$ such that $\sigma^{p} \not \tau^{p}$. The order of $\sigma^{p}$ and $\tau^{p}$ is $m / p$, which has shorter prime decomposition than $m$, so by the induction hypothesis there exists some $k$ for which $\sigma^{p k}$ and $\tau^{p k}$ are almost similar.

Having proved this, we now have a criterion to check whether a representation unites classes: It is sufficient to show that all pairs of almost similar elements remain non united, i.e. that the character of the representation takes different values on them.

Lemma 3.7. Let $\sigma$ and $\tau$ be almost similar permutations in $S_{n}$, of order $m$.

(1) If fix $(\sigma)=\operatorname{fix}(\tau)$, then $\sigma \sim \tau$.

(2) For any prime $p|m, p|(\operatorname{fix}(\sigma)-\operatorname{fix}(\tau))$.

Proof.

(1) Suppose that $\operatorname{fix}(\sigma)=\operatorname{fix}(\tau)$. We shall show that $c_{k}(\sigma)=c_{k}(\tau)$, for all $k \mid m$, by induction on the length of $k$ 's prime decomposition. We have $c_{1}\left(\sigma^{k}\right)=c_{1}\left(\tau^{k}\right)$. If $k$ is prime, then $c_{1}\left(\sigma^{k}\right)=k c_{k}(\sigma)+c_{1}(\sigma)$, so after clearing sides, we have $c_{k}(\sigma)=c_{k}(\tau)$. In the general case, we have $c_{1}\left(\sigma^{k}\right)=$ $k c_{k}(\sigma)+\sum_{t \mid k, t \neq k} t c_{t}(\sigma)$. By the induction hypothesis, the summands under the summation sign are equal for $\sigma$ and $\tau$, and so is the left hand side. This implies that $c_{k}(\sigma)=c_{k}(\tau)$.

(2) Let $p \mid m$. Then $c_{1}\left(\sigma^{p}\right)=c_{1}\left(\tau^{p}\right)$, so $p c_{p}(\sigma)+c_{1}(\sigma)=p c_{p}(\tau)+c_{1}(\tau)$. Clearing sides gives $c_{1}(\sigma)-c_{1}(\tau)=p\left(c_{p}(\tau)-c_{p}(\sigma)\right)$.

We are now in a position to re-prove the theorem on the natural representation from Section 2 over $\mathbb{C}$ :

Theorem 3.8. The natural representation $\rho_{1}: S_{n} \rightarrow G L(n, \mathbb{C})$ does not unite conjugacy classes. 
Proof. In this case $\chi(\sigma)=c_{1}(\sigma)$. Suppose $\sigma$ and $\tau$ are almost similar and $\chi(\sigma)=$ $\chi(\tau)$. This means that $c_{1}(\sigma)=c_{1}(\tau)$, and Lemma 3.7 implies that $\sigma \sim \tau$.

Using a similar technique, we can go further:

Theorem 3.9. The representation $\rho_{2}$ of $S_{n}$ on ordered pairs does not unite classes either.

Proof. In this case $\chi(\sigma)=c_{1}(\sigma)\left(c_{1}(\sigma)-1\right)$, because each fixed point of $T(\sigma)$ comes from an ordered pair of fixed points of $\sigma$. Suppose there exists a pair $\sigma$ and $\tau$ of almost similar elements of order $m$ which are united by $T$, i.e. $\chi(\sigma)=\chi(\tau)$. For convenience, denote $c_{1}(\sigma)$ by $x$ and $c_{1}(\tau)$ by $y$, and assume that $x \geq y$. Using this terminology, we have $x(x-1)=y(y-1)$. This equality can hold for integers only if $x, y \in\{0,1\}$, so $x-y<2$. However we have $p \mid(x-y)$ for any prime $p \mid m$. Since $x-y<2$, we must accept that $x=y$, and so, again by Lemma 3.7 $\sigma \sim \tau$.

It's in generalizing to triplets that we first run into trouble.

Theorem 3.10. The representation $\rho_{3}$ of $S_{n}$ on ordered triplets unites classes if and only if $n$ is even.

Proof. Here $\chi(\sigma)=c_{1}(\sigma)\left(c_{1}(\sigma)-1\right)\left(c_{1}(\sigma)-2\right)$, because each fixed point of $T(\sigma)$ comes from an ordered triplet of fixed points of $\sigma$. Let $\sigma$ and $\tau$ be a pair of almost similar elements of order $m$ which get united by the representation. Again, let $c_{1}(\sigma)=x$ and $c_{1}(\tau)=y$ and suppose $x \geq y$. We are facing $x(x-1)(x-2)=$ $y(y-1)(y-2)$. This can hold for nonnegative integers only if $x, y \in\{0,1,2\}$, so $x-y<3$.

If $n$ is even, then consider the following elements: $\sigma=(1,2)(3,4) \cdots(n-1, n)$ and $\tau=(1)(2)(3,4) \cdots(n-1, n)$. The orders are prime, so they are almost similar. Here $c_{1}(\sigma)=0$ and $c_{1}(\tau)=2$, so $\chi(\sigma)=\chi(\tau)$, and they unite under $T$.

However, if $n$ is odd, we cannot find such an example.

- If $m$ has some odd prime component $p$, then as above $p \mid(x-y)$. Since $x-y<3$, we must accept that $x=y$, which implies (Lemma 3.7 again) that $\sigma \sim \tau$.

- If $m$ is a power of 2. In this case both $\sigma$ and $\tau$ must have at least one fixed point, i.e. $x, y \geq 1$, because in $n=c_{1}\left(\sigma^{m}\right)=\sum_{k \mid m, k \neq 1} k c_{k}(\sigma)+c_{1}(\sigma)$, the left hand side is odd, and all the summands under the summation sign are even. Also, we have $2 \mid x-y$. So, if $x-y \neq 0$, then $x-y \geq 2$. This imples that $x \geq 2+y \geq 3$, contrary to our assumption about $x$ and $y$. Therefore, we must agree that $x=y$, and so that $\sigma \sim \tau$.

Theorem 3.11. The representation of $S_{n}$ on $k$-tuples for $k \geq 4$ always unites some classes.

Proof. If $n$ is even, then $\sigma=(1,2)(3,4) \cdots(n-1, n)$ and $\tau=(1)(2)(3,4) \cdots(n-$ $1, n)$ furnish an example of elements that unite, because $c_{1}(\sigma)=0$ and $c_{1}(\tau)=2$, so $\chi(\sigma)=\chi(\tau)$. Similarly, when $n$ is odd: $\sigma=(1,2)(3,4) \cdots(n-2, n-1)$ and $\tau=(1)(2)(3,4) \cdots(n-2, n-1)$ are united, because $c_{1}(\sigma)=1$ and $c_{1}(\tau)=3$, so $\chi(\sigma)=\chi(\tau)$.

In summary, we have shown that the natural representation of $S_{n}$, i.e., the representation arising from the action of $S_{n}$ on 1-tuples, does not unite classes. The 
same applies to the representation arising from the action on pairs. On triplets it works only when $n$ is odd, and beyond that, all representations unite some classes.

3.2. General Fields. The proofs in the previous section apply only to the complex field $\mathbb{C}$, (in fact, to all fields with characteristic 0.) We shall now show that the same applies to any field. We shall base ourselves on Theorem 2.2 from Section 2 where we proved that the natural representation does not unite classes, regardless the base field.

Lemma 3.12. Let $f: G \rightarrow H$ and $g: H \rightarrow K$ be group homomorphisms.

(1) If $f$ and $g$ both do not unite classes, then also $g f$ does not unite them.

(2) If $g f$ does not unite classes, then neither does $f$.

Proof.

(1) Suppose that $g f(\sigma) \sim g f(\tau)$. This implies that $f(\sigma) \sim f(\tau)$, because $g$ does not unite classes. This in turn implies that $\sigma \sim \tau$.

(2) Suppose that $f(\sigma) \sim f(\tau)$. So obviously $g f(\sigma) \sim g f(\tau)$, and since $g f$ does not unite classes, we conclude that $\sigma \sim \tau$.

Theorem 3.13. Let $T$ be any permutation representation of $S_{n}$. If $T$ does not unite classes when considered a representation into $G L(m, \mathbb{C})$, then it does not unite classes when considered as a representation into $G L(m, \mathbb{F})$, for any field $\mathbb{F}$.

Proof. Any permutation representation can be factored into $S_{n} \rightarrow S_{m} \rightarrow G L(m, \mathbb{C})$, where the first homomorphism is the permutation representation and the second is the natural representation. Now, suppose $T$ does not unite classes. By Lemma 3.12 neither does the permutation representation $S_{n} \rightarrow S_{m}$. We already know that the natural representation does not unite classes, whatever the field. Tacking these two homomorphisms together gives us the representation in any field, and another appeal to Lemma 3.12 proves that it still doesn't unite any classes.

\section{The aCtion of $S_{n} \times S_{n}$ ON invertible matrices}

In this section we present and application of Theorem 2.2

Definition 4.1. Let $\mathbb{F}$ be any field. We define an action of $S_{n} \times S_{n}$ on the group $G L(n, \mathbb{F})$ by

$$
(\pi, \sigma) \bullet A=\pi A \sigma^{-1} \text { where }(\pi, \sigma) \in S_{n} \times S_{n} \text { and } A \in G L(n, \mathbb{F})
$$

It is a group action since:

$$
\begin{aligned}
& (e, e) \bullet A=e A e=A \\
& \qquad \begin{aligned}
\left(\pi_{1}, \sigma_{1}\right) & \bullet\left(\left(\pi_{2}, \sigma_{2}\right) \bullet A\right)=\left(\pi_{1}, \sigma_{1}\right) \bullet\left(\pi_{2} A \sigma_{2}^{-1}\right)= \\
& \pi_{1} \pi_{2} A \sigma_{2}^{-1} \sigma_{1}^{-1}=\left(\pi_{1} \pi_{2}, \sigma_{1} \sigma_{2}\right) \bullet A=\left(\left(\pi_{1}, \sigma_{1}\right)\left(\pi_{2}, \sigma_{2}\right)\right) \bullet A
\end{aligned}
\end{aligned}
$$

Definition 4.2. Let $M$ be a finite subset of $G L(n, \mathbb{F})$, invariant under the action of $S_{n} \times S_{n}$ defined above. We denote by $\alpha_{M}$ the permutation representation of $S_{n} \times S_{n}$ obtained from the action (1). In the sequel we identify the action (1) with the permutation representation $\alpha_{M}$ associated with it. 
Now we define a generalization of the conjugacy representation of $S_{n}$

We present a conjugacy representation of $S_{n}$ on a subset $M$ of $G L(n, \mathbb{F})$.

Definition 4.3. Denote by $\beta$ the permutation representation of $S_{n}$ obtained by the following action on $M$.

$$
\pi \circ A=(\pi, \pi) \bullet A=\pi A \pi^{-1}
$$

The connection between $\alpha_{M}$ and $\beta_{M}$ is given by the following easily seen claim:

Claim 4.4. Consider the diagonal embedding of $S_{n}$ into $S_{n} \times S_{n}$. Then

$$
\beta_{M}=\alpha_{M} \downarrow_{S_{n}}^{S_{n} \times S_{n}} .
$$

Theorem 4.5. For every finite set $M \subseteq G L(n, \mathbb{F})$ invariant under the action (1) of $S_{n} \times S_{n}$ defined above:

If $\pi$ and $\sigma$ are conjugate in $S_{n}$ then

$$
\chi_{\alpha_{M}}((\pi, \sigma))=\chi_{\alpha_{M}}((\pi, \pi))=\chi_{\beta_{M}}(\pi)=\#\{A \in M \mid \pi A=A \pi\} .
$$

If $\pi$ is not conjugate to $\sigma$ in $S_{n}$ then

$$
\chi_{\alpha_{M}}((\pi, \sigma))=0 .
$$

Proof. If $\pi$ and $\sigma$ are conjugate in $S_{n}$ then $(\pi, \sigma)$ is conjugate to $(\pi, \pi)$ in $S_{n} \times S_{n}$. Since the character is a class function, we have:

$$
\chi_{\alpha_{M}}(\pi, \sigma)=\chi_{\alpha_{M}}(\pi, \pi)=\#\left\{A \in M \mid \pi A \pi^{-1}=A\right\}=\#\{A \in M \mid \pi A=A \pi\}
$$

i.e. the value of the character of $\alpha_{M}$ calculated on the element $(\pi, \sigma)$ with $\pi$ conjugate to $\sigma$ in $S_{n}$ is equal to the number of matrices in $M$ which commute with the permutation matrix $\pi$.

Now, we know that the character of a permutation representation counts the number of fixed points, so:

$$
\chi_{\alpha_{M}}(\pi, \sigma)=\#\left\{A \in M \mid \pi A \sigma^{-1}=A\right\}=\#\left\{A \in M \mid \pi=A \sigma A^{-1}\right\} .
$$

Note that $\pi=A \sigma A^{-1}$ means that $\pi$ and $\sigma$ are similar as invertible matrices. Thus, by Theorem 2.2 if $\pi$ and $\sigma$ are not conjugate in $S_{n}$ they can not be conjugate in $G L(n, \mathbb{F})$ and we have:

$$
\left\{A \in M \mid \pi=A \sigma A^{-1}\right\}=\varnothing
$$

and so

$$
\chi_{\alpha_{M}}(\pi, \sigma)=0
$$

if $\pi$ and $\sigma$ are not conjugate in $S_{n}$. This result is applied in [BC].

Acknowledgments. The authors are grateful to Alex Lubotzky, Yuval Roichman, Eli Bagno, Uzi Vishne and Boris Kunyavskii for helpful discussions.

\section{REFERENCES}

[B] William Burnside, Theory of Groups of Finite Order. University Press, Cambridge 1897.

[BC] Eli Bagno and Yona Cherniavsky, Permutation representations on invertible matrices. Arxiv reference math.RT/0411556 To be published.

[CR] Charles Curtis and Irving Reiner, Representation Theory of Finite Groups and Associative Algebras. John Wiley and Sons, New York 1962.

[FH] William Fulton and Joe Harris, Representation Theory, a First Course. Springer, New York 1991

[S] Bruce Sagan, The Symmetric Group. Representations, Combinatorial Algorithms, and Symmetric Functions. Springer, New York 2001 
[Se] Jean-Pierre Serre, Linear Representations of Finite Groups. Springer, New York 1977

Department of Mathematics, Bar Ilan University, Ramat Gan 52900, Israel

E-mail address: cherniy@math.biu.ac.il

Department of Mathematics, Bar Ilan University, Ramat Gan 52900, Israel

E-mail address: sklarzm@math.biu.ac.il 